\title{
El pensamiento social cristiano salvadoreño: el caso de Monseñor José Alfonso Belloso y Sánchez (1927-1935).
}

René Chanta Martínez ${ }^{1}$

Recibido en Marzo 2012, aprobado en Agosto 2012

\section{Resumen.}

Este artículo muestra algunos de los temas sociales abordados por Monseñor José Alfonso Belloso y Sánchez entre 1927 y 1935 en sus Cartas Pastorales, cuando dirigió la Arquidiócesis de San Salvador. Estos temas hacen especial énfasis en la violencia y el anticomunismo. Este último, cobra especial relevancia por los acontecimientos ocurridos por el levantamiento de 1932.

\section{Palabras clave:}

Pensamiento Social Cristiano, El Salvador, anticomunismo, violencia, Monseñor Belloso y Sánchez.

\footnotetext{
Abstract

This article shows some of the social issues addressed by Bishop José Alfonso Belloso y Sánchez between 1927 and 1935 in his Pastoral Letters, when he led the Archdiocese of San Salvador. These topics place particular emphasis on violence and anti-communism. The latter is particularly relevant for the happenings of the 1932 uprising.

\section{Keywords}

Social christian thinking, El Salvador, Anti-communism, Violence, Monsignor Belloso y Sánchez
}

\section{Introducción.}

Los estudios sobre la reflexión social de los cristianos en los primeros años del siglo XX no son muy frecuentes en El Salvador. En efecto, muchas investigaciones ${ }^{2}$ se han centrado casi exclusivamente en los últimos treinta años del recién pasado siglo, en donde se dio de una manera acentuada el conflicto entre los

\footnotetext{
1 Profesor de Pensamiento social cristiano, en la Universidad Don Bosco.

E-mail: rene.chanta@udb.edu.sv

2 Cfr. Rodolfo Cardenal, (1987) Historia de una esperanza, vida de Rutilio Grande, UCA editores, Segunda edición, San Salvador; Jesús Delgado (1994), Oscar A. Romero, biografía, UCA editores, San Salvador; Juan Vicente Chopin (2010), La Iglesia de los mártires, una lectura latinoamericana desde El Salvador y Guatemala (1977-1998), Editorial Don Bosco, San Salvador.
} 
sectores gubernamentales y diversas entidades cristianas, produciéndose una fuerte represión estatal y paraestatal ${ }^{3}$. En esos años, sobretodo la década de los setenta e inicios de los ochenta, fue época muy rica a nivel martirial, ya que personas como Monseñor Romero, Rutilio Grande, Alfonso Navarro, entre otros, dieron su vida en estos tiempos conflictivos. A la vez, varios personajes cristianos nos legaron sus reflexiones en diversos documentos escritos que hoy forman una especie de patrimonio del pensamiento social cristiano salvadoreño.

Por el contrario, los primeros años del siglo XX han estado hasta cierto punto marginados de los estudios y análisis teológicos. Sin embargo, al leer diversos impresos católicos de las primeras décadas del siglo XX, se puede constatar que los problemas sociales, que siempre han estado presentes en nuestro país, han sido afrontados por la Iglesia, que se ha pronunciado contra las injusticias, ya desde esos años. Por otro lado, conviene decir que también hay en los archivos ${ }^{4}$, varias Cartas Pastorales con fuerte contenido social, escritas por los diferentes obispos que tenían al frente las diócesis del territorio salvadoreño en esa época. En ese sentido, el objetivo del presente ensayo es realizar un acercamiento al Pensamiento Social Cristiano que fue escrito por el segundo Arzobispo de San Salvador: José Alfonso Belloso y Sánchez. Conviene advertir que no se va a pretender hacer una exposición y análisis exhaustivo, que agote y dé cuenta de cada uno de los escritos de este arzobispo, ya que para eso haría falta un estudio más prolongado y detallado. Más bien, en este trabajo se procederá a hacer una selección de algunos de los textos de Belloso y Sánchez, en donde se abordan prioritariamente los temas políticos y sociales que afectaban la realidad nacional e internacional en el período de tiempo comprendido entre los años de 1927 y 1935.

El ministerio pastoral de Belloso y Sánchez fue hasta cierto punto, un periodo largo temporalmente ${ }^{5}$. Su mandato episcopal lo realizó desde 1920 hasta $1939^{6}$.

3 El conflicto social no solo se dio a grupos cristianos sino que abarcó a diversas organizaciones sociales. En efecto, la fuerte represión de los sectores estatales culminó con una cruenta guerra civil que dejó más de 70.000 muertos.

4. Este trabajo forma parte de una investigación más amplia que he estado realizando sobre el Pensamiento Social Cristiano Salvadoreño en el siglo XX. Resalto que el principal lugar de investigación ha sido el Archivo Histórico del Arzobispado de San Salvador, entidad que me ha facilitado en gran medida diversas fuentes primarias.

5. Evidentemente, no se puede decir que su periodo haya sido de los más largos en la Arquidiócesis de San Salvador. Otros obispos han tenido ministerios pastorales bastante más extensos. Por ejemplo, Chávez y González duró casi cuarenta años al frente de la Iglesia de San Salvador. Al respecto, consúltese: Rosa Carmelita Samos, Sobre el magisterio de Mons. Luis Chávez y González, Estudio teológico de sus cartas pastorales, Tesis de Licenciatura en Teología, Universidad Francisco Marroquín, Guatemala, julio de 1986.

6. En este aspecto hay que destacar que durante este periodo de tiempo, no siempre fue Arzobispo. Fue consagrado obispo en 1920, convirtiéndose en obispo auxiliar de San Salvador. Luego, cuando quedó vacante la arquidiócesis, fue durante un año su Administrador Apostólico. En ese año lanzó una Carta Pastoral titulada "El Presente Momento Social". Fue hasta agosto de ese que fue nombrado Arzobispo de San Salvador. Cfr. Ramón López Jiménez, Mitras Salvadoreñas, Publicaciones del Ministerio de Educación, San Salvador, 1960, págs. 141-177. 
Como puede verse, en total fueron 19 años al frente de la Iglesia capitalina, tiempo durante el cual se llevaran a cabo algunos acontecimientos que marcaron el futuro del país: el levantamiento campesino de 1932, el inicio de los mandatos militares de Maximiliano Hernández Martínez, etc. Belloso y Sánchez, con sus cartas pastorales, dará respuesta a este contexto local. A la vez, conviene tener en cuenta otros hechos y sucesos internacionales que van a influir sobre la situación nacional: la crisis económica de 1929, las secuelas de la primera guerra mundial, el avance del comunismo sobre Europa, entre otros temas.

Teniendo en cuenta lo anterior, este trabajo tendrá tres partes. En un primer momento se explicará de manera breve qué es lo que se va a entender por Pensamiento Social Cristiano. Esto nos dará una base teórica para presentar esta investigación. Luego, se hará un acercamiento a los datos biográficos de Monseñor Belloso y Sánchez, resaltando el contexto social en el que desarrolló su ministerio pastoral. Finalmente, se mostrarán algunos escritos de este arzobispo salvadoreño, en donde muestra una gran sensibilidad hacia los temas sociales y políticos.

\section{1. ¿Qué es Pensamiento Social Cristiano?}

Antes de entrar a ver detalladamente los textos emitidos por Belloso y Sánchez conviene hacer una pequeña aproximación al significado del término "Pensamiento Social Cristiano". Esto es de suma importancia puesto que en los ambientes académicos y pastorales se habla continuamente de Doctrina Social de la iglesia, Pensamiento Social cristiano, Doctrina Social Cristiana y Enseñanza Social Cristiana. En ese sentido, podríamos hacernos la siguiente pregunta: ¿es lo mismo Pensamiento Social Cristiano que Doctrina Social de la Iglesia?

\section{1 ¿Qué es pensamiento social cristiano?}

Primeramente, hay que decir que no es fácil dar una definición de Pensamiento Social Cristiano. El término más utilizado en ambientes eclesiales es Doctrina Social de la Iglesia. En efecto, hoy en día existen muchas publicaciones en donde prevalece el uso de Doctrina Social Cristiana en lugar de Pensamiento Social Cristiano. Históricamente, se considera que el primer documento de la Iglesia sobre Doctrina Social es la encíclica Rerum Novarum ${ }^{7}$, publicada en el año de 1891 , en donde se aborda sobretodo la cuestión obrera. Curiosamente, en esta encíclica papal nunca se utilizó el vocablo “Doctrina Social de la Iglesia”. Lo anterior, obliga al investigador a rastrear en los diversos textos papales cómo este vocablo fue ganando fuerza hasta llegar a imponerse como un término de uso normal.

7. Encíclica publicada por el Papa León XIII en 1891 y cuyo tema central es la cuestión obrera. Disponible en línea: http://www.vatican.va/holy_father/leo_xiii/encyclicals/documents/hf_lxiii_enc_15051891_rerum-novarum_sp.html (Consultado el 5 de abril de 2012). 
El término Doctrina Social de la Iglesia es una expresión que aparece ya entrado el siglo XX y que el Papa Pio XII usó frecuentemente. Sin embargo, otros papas no utilizan ese vocablo. Por ejemplo, Pablo VI prefiere utilizar otros términos como "mensaje" u "orientaciones". Ya con Juan Pablo II, Doctrina Social de la Iglesia vuelve a ser mencionado con mucha frecuencia. Ejemplos de esto han quedado evidenciados en el Discurso de apertura de Puebla ${ }^{8}$ y en sus propios escritos sobre temas sociales como Laborem et Excercens ${ }^{9}$ y Sollicitudo Rei Socialis ${ }^{10}$.

Por su parte, el magisterio latinoamericano, en sus diversos escritos, utiliza expresiones como Enseñanza Social de la Iglesia. Por ejemplo, el texto de Puebla en el número 472, nos dice: “el aporte de la Iglesia a la liberación y promoción humana se ha venido concretando en un conjunto de orientaciones doctrinales y criterios de acción que solemos llamar "enseñanza social de la Iglesia ${ }^{11 "}$.

Por otro lado, no podemos dejar de lado el hecho de que la reflexión teológica ha sido fecunda a la hora de analizar las temáticas sociales. En ese sentido, múltiples teólogos han dado su definición de lo que se entiende por Doctrina Social de la Iglesia. Por ejemplo, Kerber la define como "la formulación del mensaje del evangelio ante las múltiples y cambiantes realidades sociales ${ }^{12}$ ". Esta definición es muy significativa ya que muestra que a pesar de que en el cristianismo hay puntos de referencia inamovibles, también se hace necesario actualizar ese mensaje evangélico ante los diversos contextos en los que se profesa la fe.

A pesar de la gran riqueza conceptual de las definiciones anteriores al hablar sobre Doctrina Social de la Iglesia, para algunas personas, la palabra "Doctrina" es algo desfasado o desactualizado ya que muestra una visión muy pasiva de las personas creyentes. Así, ya desde la década de los años cincuenta había dentro del catolicismo un gran movimiento de renovación litúrgica, exegética y teológica. Muchos de estos puntos de vista distintos y más renovados desembocarían en lo que se plasmó en el Concilio Vaticano II. También, por estos años se dio una fuerte crítica a muchos vocablos usados en los ambientes teológicos y pastorales. Uno de esos términos que fue blanco de críticas y

8. Tercera Conferencia del Episcopado Latinoamericano (CELAM) celebrada en 1979 en la ciudad de Puebla, México.

9. En ocasión de los noventa años de haber salido a la luz el texto de Rerum Novarum, el Papa Juan Pablo II, escribió una encíclica titulada Laborem Excercens (El Trabajo ejercido). Se publicó en septiembre de 1981. Aquí nuevamente vuelve el magisterio eclesial a tratar el tema del trabajo como clave de la cuestión social.

10. Vergara, Exequiel Rivas y otros, Manual de Doctrina Social de la Iglesia, Colección de textos básicos para Seminarios, Bogotá, Colombia, 2001.

11. Tercera Conferencia del Episcopado Latinoamericano, Puebla, 1979.

12. Manual de Doctrina Social Cristiana, Instituto Mexicano de Doctrina Social de la Iglesia, 1991, pág. 22 
sospechas fue Doctrina. En ese sentido, a criterio de algunos, Doctrina era un vocablo que daba la sensación de referirse a situaciones estáticas, rígidas e inamovibles. Es por eso que muchos pensadores han ido a la búsqueda de otras palabras que expliquen más coherentemente el conjunto de enseñanzas de la Iglesia en torno a las temáticas sociales.

Una de las palabras que más han ido calando en la conciencia de los sectores católicos es el de Pensamiento Social Cristiano, para referirnos a toda la serie de escritos, documentos y palabras producidas por los grupos cristianos para analizar los temas sociales desde la fe. En efecto, a juicio de algunos, el concepto "Pensamiento" da una sensación de apertura y dinamicidad contrario al estático y rígido concepto “Doctrina”.

En base a todo lo anterior, podríamos decir que en sentido general, Pensamiento Social Cristiano y Doctrina Social de la Iglesia, se pueden utilizar como expresiones equivalentes. En este punto, un texto nos dice que si bien es cierto el término doctrina social cristiana ha sido un constructo del siglo XX, puede ser sustituido por expresiones sinónimas. Para fines de este trabajo, aquí se preferirá el uso del término Pensamiento Social Cristiano porque esa palabra permite tener una visión más amplia y dinámica de lo que los sectores cristianos han reflexionado en torno a los temas sociales y políticos.

\subsection{Pensamiento Social Cristiano en sentido estricto y en sentido amplio.}

Tal como hemos dicho líneas atrás, a lo largo de este trabajo se utilizará únicamente el término Pensamiento Social Cristiano para hacer alusión a las reflexiones de personas creyentes sobre temáticas sociales. Sin embargo, es conveniente ganar precisión sobre lo que estamos entendiendo por Pensamiento Social Cristiano. A mi modo de ver, habría dos grandes modos de aproximarse al término Pensamiento Social Cristiano: uno en sentido estricto y otro en sentido amplio.

En sentido estricto, podríamos decir que Pensamiento Social Cristiano se refiere al conjunto de textos, pronunciamientos y escritos realizados por miembros de la jerarquía eclesiástica (papas, obispos, sacerdotes) en los que se abordan diferentes problemáticas sociales. Estos problemas sociales se iluminan y se juzgan desde la fe cristiana para dar principios orientadores para la acción. Aunque siempre en el cristianismo han existido discursos y escritos sobre problemas sociales por parte de los prelados católicos, en el mundo contemporáneo la Encíclica Rerum Novarum marcó un hito al ser considerada el primer texto de Pensamiento Social Cristiano. Desde este documento escrito en 1891 hasta la actualidad, han pasado más de cien años y se ha producido un considerable número de encíclicas, cartas pastorales, circulares y otros documentos de pensamiento social cristiano emitidos por la suprema autoridad 
católica: el Papa. Entre los documentos papales se encuentran Quadragesimo $a_{n n o}{ }^{13}$, Sollicitudo rei socialis, Pacem in Terris, Centesimus annus, etc.

Al mismo tiempo, no hay que olvidar que a nivel latinoamericano, los análisis y reflexiones sobre temas sociales han sido muy significativos e importantes. Solo por ejemplificar lo anterior, se puede hacer alusión a los documentos de Medellín, de Puebla, Santo Domingo y, más recientemente, al documento de Aparecida. En estos textos, los obispos, viendo las particularidades propias de América Latina, procederán a juzgar desde la fe cristiana las situaciones injustas que vive la región.

Por otra parte, en un sentido más extendido, el término Pensamiento Social Cristiano abarcaría no solamente las reflexiones emitidas por los clérigos, sino que también recoge el pensar de todas las personas creyentes que desde los parámetros cristianos emiten sus reflexiones sobre los problemas sociales que vive su contexto determinado (ciudad, país, barrio, etc). Lo anterior ocurre porque un cristiano no puede estar desconectado de la realidad social en la que vive. Se podría decir que es un imperativo cristiano trabajar por un mundo más justo y más equitativo, anunciando lo correcto y denunciando la injusticia. No hacerlo sería ir contra la lógica de la encarnación.

\section{Breves apuntes biográficos sobre José Alfonso Belloso y Sánchez.}

José Alfonso Belloso y Sánchez, nació en la capital salvadoreña, el 30 de octubre de 1873. Realizó sus estudios teológicos en Roma y se ordenó sacerdote en 1897, cuando tenía 24 años de edad. Cuando regresó al país realizó diversas tareas pastorales, como capellán del Ejército Salvadoreño, párroco en Suchitoto y Ahuachapán. En 1919, el Papa Benedicto XV lo nombró obispo auxiliar de San Salvador ${ }^{14}$. En 1926 la sede episcopal de San Salvador quedó vacante ante el deceso de Monseñor Adolfo Pérez y Aguilar ${ }^{15}$. Ante esto, un año después, el Papa nombró a Belloso y Sánchez como nuevo Arzobispo de San Salvador. Murió el 9 de agosto de 1938.

Durante su labor pastoral redactó varias cartas pastorales abarcando muchos temas entre los que sobresalen temas litúrgicos, morales, eclesiales y sociales. Su ministerio pastoral en San Salvador coincidió con los gobiernos de la 'dinastía'

$\overline{\text { 13. Encíclica publicada en } 1931 \text { por el Papa Pio XI, en ocasión del cuarenta aniversario de la Rerum }}$ Novarum.

14. Cfr. Ramón López Jiménez, Mitras Salvadoreñas, Publicaciones del Ministerio de Educación, San Salvador, El Salvador, 1960, págs. 141-145

15. Monseñor Pérez y Aguilar gobernó la Iglesia de San Salvador por un espacio de 38 años. Durante su mandato episcopal, Su Santidad Pio X erigió la Provincia Eclesiástica de El Salvador en 1913 con el documento "Americae Centralis". De esta manera, San Salvador pasó a ser la diócesis Metropolitana y su titular se convirtió en el primer Arzobispo. Esta Iglesia Metropolitana tenía dos diócesis sufragáneas: Santa Ana y San Miguel. Pérez y Aguilar falleció a la edad de 87 años. Para más datos, consúltese, Estatutos del Venerable Cabildo Eclesiástico de la Santa Iglesia Catedral Metropolitana, Tipografía La Unión, San Salvador, El Salvador, 1942, pág. 4. 
Meléndez Quiñonez, Arturo Araujo y Maximiliano Hernández Martínez. Los ocho años comprendidos entre 1927 y 1935 fueron muy significativos para la historia salvadoreña, ya que durante ese periodo de tiempo se dieron sucesos insólitos en la vida nacional. Por ejemplo, se vivió un golpe de Estado realizado por los sectores militares. Este acontecimiento produjo el inicio de una de las dictaduras más largas en el país, la de Maximiliano Hernández Martínez, y la consolidación del militarismo. Esto trajo graves consecuencias ya que se rompió con un ciclo de sucesión de gobernantes cada cuatro años, tal como mandaba la Constitución de entonces ${ }^{16}$.

A lo anterior hay que agregar que el modelo liberal, impulsado en diversas naciones centroamericanas desde mediados del siglo XIX, había entrado en crisis $^{17}$. Esto se agravó con la crisis económica mundial de 1929. En el caso de las naciones del istmo centroamericano, los efectos de la crisis empezaron a sentirse con el descenso del precio de las exportaciones. En El Salvador este descenso fue de casi el 60 por ciento. Según Rodolfo Cardenal, la respuesta más clara realizada por muchos países ante la crisis del modelo liberal fue instaurar dictaduras militares. De hecho, los vecinos países a excepción de Costa Rica, formaron dictaduras.

Finalmente, en enero de 1932 se dio un levantamiento en El Salvador en donde miles de indígenas y campesinos en el occidente del territorio nacional atacaron puestos policiales, cuarteles militares y ciertos pueblos. A juzgar por las referencias, este levantamiento tenía elementos del pensamiento comunista. Al respecto, Thomas Anderson muestra que desde la campaña electoral de Araujo ya había un acelerado incremento de ideas de extrema izquierda en el territorio salvadoreño. De acuerdo a este mismo autor, el movimiento de 1932 aunque tenía influencias del comunismo, fue casi exclusivamente un movimiento autónomo en donde sus líderes actuaron por su propia cuenta (Farabundo Martí, Miguel Mármol, Rafael Bondanza, entre otros) ${ }^{18}$. Esto produjo una fuerte represión estatal a través del ejército y la Guardia Nacional, que dejó como saldo incontables muertos. Esto es lo que se conoce como 'la matanza de 1932'. Ese fue el contexto que le tocó enfrentar a Monseñor Belloso y Sánchez. Como vemos fue un momento difícil y problemático ya que fue una época de múltiples descontentos e injusticias sociales.

\footnotetext{
16. La Constitución vigente en este tiempo era la de 1886, la cual afirmaba en el artículo 82 que: "La duración del período presidencial será de cuatro años; y el ciudadano que hubiese ejercido la Presidencia en propiedad, no podrá ser reelecto ni electo vicepresidente sino después de haber transcurrido igual periodo que comenzará y concluirá el primero de marzo del año de la renovación sin poder funcionar un día más".

Cfr.http: / / bib.cervantesvirtual.com/servlet/SirveObras/01477394433725584232268/p0000001. htm\#l_11_ (consultado el 5 de abril de 2012).

17. Cfr. Rodolfo Cardenal, Manual de Historia de Centroamérica, UCA Editores, San Salvador, 2005, págs. $341 \mathrm{ss.}$

18. Cfr. Thomas Anderson, El Salvador, 1932, CONCULTURA, San Salvador, 2001, págs. 159-179.
} 


\section{Algunos escritos sociales de Monseñor Belloso y Sánchez.}

A continuación presentaré algunos escritos de Monseñor Belloso y Sánchez, en los cuales abordó algunos temas sociales de gran envergadura. Sería imposible en este espacio abordar todos los temas a los que se refirió este arzobispo. Me limitaré solamente a dos problemática: el alto índice de violencia y el anticomunismo ${ }^{19}$.

\subsection{Sobre la violencia.}

Un texto de Belloso y Sánchez en el que trata ampliamente sobre la violencia es la Sexta Carta Pastoral, que se titula "Non occides, No matarás (Éxodo $X X-13)^{20}$ ". Aquí mostrará de forma detallada que la vida humana es sagrada porque viene de Dios y que todo homicidio es un crimen. Por otro lado, también evidencia que uno de los grandes causantes de las muertes en el país es el alcoholismo. Nos dice: “¿quién ignora que adolece nuestra sociedad de otra plaga no menos espantable: el alcoholismo, y que el alcoholismo es una de las causas más eficaces de los crímenes de sangre? ${ }^{21 "}$.

En este mismo documento, Monseñor Belloso y Sánchez señala que otra causa de las muertes en el país es el alto número de personas que portan armas. Esto influye en el alto número de homicidios y suicidios. Lo peor de esto es, a juicio de esta sexta carta pastoral, la combinación del alcohol con las armas. Finalmente, el Arzobispo de San Salvador hace un fuerte llamado a defender la vida humana. Dice Belloso y Sánchez:

nunca deja el pulcro caballero la primorosa escuadra ni el modesto burgués el revolver vulgar, ni el hombrecito del campo su tosco machete. Amados hijos: defender la vida, bien está; pero haberla de mantener a salvo con el uso continuo de las armas desdice de un pueblo culto y cristiano [...]. Gana ciertamente el fisco otorgando licencia de ir armados, mas la religión y la paz y la cultura y la suavidad de costumbres y la humanidad misma, pierde. ${ }^{22}$

Como vemos, esta carta pastoral no sólo critica al ciudadano común y corriente por tener una cultura no muy acorde a los valores cristianos, sino que también arremete contra el gobierno al hacerlo partícipe indirecto de estas muertes, al lucrarse de los significativos impuestos que llegan a sus arcas como producto del control y reglamentación de las armas de fuego. Por otro lado, también nos muestra que la realidad salvadoreña siempre ha tenido altos índices de

19. Ciertamente hubo otros temas sociales abordados en sus Cartas Pastorales. Por ejemplo, la decimoquinta carta pastoral está dedicada a las víctimas del temporal de junio de 1934 .

20. Todas las referencias que aquí se hagan de las Cartas Pastorales de Monseñor José Alfonso Belloso y Sánchez han sido tomadas del Archivo Histórico del Arzobispado de San Salvador (AHAS). 21. "No matarás", Sexta Carta Pastoral de Monseñor Belloso y Sánchez", San Salvador, 6 de agosto de 1930, pág 2.

22. Ibíd., pág 3. 
violencia. Esto último es muy significativo, ya que en la actualidad El Salvador posee altos índices de homicidios producidos sobretodo con armas de fuego. Por otra parte, es muy interesante el vínculo que hace Belloso y Sánchez entre alcoholismo y violencia. Esto también es de mucha actualidad ya que hoy en día el alcoholismo produce violencia intrafamiliar y múltiples catástrofes como los accidentes automovilísticos. Finalmente, este texto citado desenmascara algo muy cierto: el gran negocio que producen las armas. En este punto, es claro que el gobierno gana al poner impuesto a las armas, pero también hay muchas empresas lucrándose de estos artefactos.

\subsection{Sobre el comunismo.}

Otro de los temas comentados en las Cartas Pastorales de Belloso y Sánchez fue el comunismo, que él veía como una amenaza para el territorio nacional. Así en 1927, Belloso y Sánchez publicó el texto "Pastoral del administrador apostólico de San Salvador sobre el presente momento social”. El documento comenzaba lamentándose por las diversas publicaciones en la prensa y hojas volantes que difundían las ideas socialistas en el país. Hace un fuerte llamado a no adherirse a los grupos socialistas. Nos dice:

pues luego un católico que se aficione a los sistemas socialistas corre grave riesgo de contaminarse de error y de herejía; riesgo más mortal, cuanto menor sea la instrucción religiosa y menos intensa la vida cristiana del aficionado ${ }^{23}$.

Por otro lado este documento proponía que se formen círculos de estudio para conocer la compleja realidad social de nuestro territorio y no adherirse a ideas socialistas venidas de regiones extranjeras. En ese sentido hace un llamado a los intelectuales del país a sumarse a este esfuerzo para encontrar soluciones concretas a los graves problemas sociales que aquejan al país ${ }^{24}$.

Al hablar de comunismo en El Salvador, no se puede pasar de largo el año 1932, año en el que se produce un levantamiento campesino en el occidente del país. Esto produjo una dura represión del gobierno encabezado por Maximiliano Hernández Martínez. Ante este suceso, los sectores cristianos no guardaron silencio. En algunos textos denunciaron las injusticias y excesos cometidos tanto por el gobierno como por los campesinos alzados. En este contexto sale a la luz la novena Carta Pastoral de Belloso y Sánchez. Este texto lleva por título "Importancia económico-social-religiosa del salario agrícola en El Salvador 25 ".

\footnotetext{
23. Belloso y Sánchez, "Pastoral del administrador apostólico de San Salvador sobre el presente momento social”, pág. 7.

24. Cfr. Ibíd., pág 22.

25. Belloso y Sánchez, Novena Carta Pastoral, “Importancia, económico-social-religiosa del salario agrícola en El Salvador".
} 
En ese texto, el Arzobispo de San Salvador lanza duras críticas a las ideas comunistas y también al acaparamiento e injusticias de los sectores más ricos y poderosos del país. La carta inicia recordando lo que él había escrito en 1927 sobre la amenaza comunista. Cinco años después y a pocos días de haberse producido el levantamiento campesino, Belloso y Sánchez afirma lo siguiente:

creemos en verdad que las doctrinas del comunismo han penetrado en cada una de las condiciones de las personas, profesiones y oficios que forman nuestras clases medias y humilde; pero han causado mayor estrago, a juzgar por los hechos, en los campesinos y colonos, quizá por ser ellos los más desfavorecidos en el orden económico. ${ }^{26}$

En efecto, el texto continúa condenando la lucha de clases. A la vez pide a los diferentes sectores involucrados en el conflicto a concertar sus intereses. Podría parecer que esta Carta Pastoral seguirá condenando el comunismo, pero no es así. Acusa a los sectores ricos de ser una de las fuentes de las injusticias en el país. Al respecto, pide a los sectores más ricos que sean justos con sus obreros e incluso pide reformas económicas concretas. Nos dice el texto:

ni vayáis a tildarnos de imprudencia porque os proponemos reformas económicas que sin duda descantillarían un tanto vuestras rentas, precisamente cuando un desfalco nunca visto trastorna hasta los cimientos el mundo de la riqueza social. La crisis económica podría convertirse en pretexto para haceros remisos y diferir a bonancibles tiempos el mejoramiento del colono [...] Podrá ser económicamente imposible el alza súbita del salario y trocar como por ensalmo la condición del labriego; pero apremia trabajar con vigor y presteza en enderezar las injusticias sociales ${ }^{27}$.

En la anterior cita, es interesante ver la reflexión del Arzobispo salvadoreño al pedir a los sectores poderosos que también pongan de su parte para resolver la situación social. Belloso y Sánchez es consciente de que dicha tarea no es fácil debido a la crisis económica de 1929, que ya se había extendido en todo el mundo y cuyos efectos habían comenzado a vislumbrarse en Centroamérica. Sin embargo, enfatiza que esa no es una excusa para no trabajar en la solución de las graves injusticias sociales. La Carta Pastoral prosigue citando continuamente las encíclicas Rerum Novarum y Quadragessimo Anno para referirse a las relaciones entre trabajo y capital, entre el obrero y el patrono y entre el trabajo y la empresa. A la vez, continúa mostrando las injusticias sociales presentes en nuestro país en el ambiente laboral. Al respecto, critica duramente cuando las empresas no pagan el salario justo. Nos dice al respecto: 
cuántas veces se consumen casi todos los productos de una finca en redimir intereses de hipotecas, en pagar impuestos exorbitantes o mal tasados [.... Más delicado se presenta el caso en que la perturbación y atraso técnico y económico de los negocios provienen de una inmoralidad -digamos- descuido y pereza, imprudencia y despilfarro del dueño o bien nacen de un defecto natural de talento y destreza. Porque peca, no contra justicia, pues dispone de lo suyo, sino contra la caridad y el deber de cooperar ${ }^{28}$.

La carta pide también que el patrono debe acrecentar la caridad con el obrero. Una manera de lograrlo es haciendo obras benéficas y educativas en favor del colono. Así, el patrono supliría lo que le falta al salario. Finalmente, el texto hace un llamamiento al Estado para que resguarde los derechos y administre correctamente la justicia ${ }^{29}$. Como podemos ver en las citas anteriores, a pesar de que el obispo es un férreo anticomunista ${ }^{30}$, también va al fondo del problema y ve que las causas del descontento de la población tienen su raíz en las injusticias cometidas por patronos y dueños. Ante lo anterior, pedirá mayores relaciones laborales justas.

\section{Reflexiones finales.}

En las páginas anteriores, hemos podido darnos cuenta de los duros años conflictivos que atravesó la realidad nacional en la segunda y tercera década del siglo XX. Se podía ver el descontento social y las graves injusticias que rodeaban las relaciones laborales. Al mismo tiempo Centroamérica veía el advenimiento de las dictaduras militares. Ante estos hechos, la Iglesia no guardó silencio. Más bien, se lanzó a denunciar los efectos negativos que tenían las decisiones gubernamentales. Ante esto se puede concluir que Monseñor Belloso y Sánchez pudo plasmar muy bien sus reflexiones pastorales sobre los temas políticos y sociales en continuo diálogo con la realidad que le rodeaba y el naciente pensamiento social cristiano que provenía de los papados.

Muchos de los temas abordados por Belloso y Sánchez son sumamente actuales. Por ejemplo, la violencia en nuestros días ha cobrado cifras alarmantes. A la vez, el tema del trabajo es muy significativo en la sociedad contemporánea, ya que desde hace unos años nuevamente se vuelve a hablar de un período de crisis económica mundial que ya ha tenido efectos negativos en países europeos como Grecia, España y Portugal. Además, en países como El Salvador, los efectos de las crisis han comenzado a experimentarse. Por ejemplo, una sociedad como la salvadoreña tiene un fuerte desempleo y subempleo de grandes sectores de la

28. Ibíd., pág 10.

29 Cfr. Ibíd., págs. 16-20

30 El tema del anticomunismo continuará en las producciones escritas de Belloso y Sánchez. En efecto, en 1937 publica otra carta pastoral "Sobre el comunismo Ateo". En esta carta da a conocer a sus diocesanos la Encíclica "Divini Redemptoris", de Pio XI. 
población. En ese sentido, a pesar de los cambios y diferentes características que tiene nuestra sociedad salvadoreña actual, las reflexiones y enseñanzas realizadas por Belloso y Sánchez, junto con otras producciones más recientes de los obispados salvadoreños, son unos referentes ineludibles que pueden iluminar reflexiones y acciones para construir una mejor sociedad.

\section{Bibliografía.}

Anderson, T, (2001), El Salvador 1932, CONCULTURA, San Salvador.

Cardenal, R, (2001), Manual de historia de Centroamérica, UCA Editores, San Salvador.

Cardenal, R, (1987), Historia de una esperanza, vida de Rutilio Grande, UCA editores, segunda edición.

Chopin, J, (2010), La Iglesia de los mártires, una lectura latinoamericana desde El Salvador y Guatemala (1977-1998), Editorial Don Bosco, San Salvador.

Delgado, J, (1994), Oscar A. Romero, biografía, UCA editores, San Salvador.

Estatutos del Venerable Cabildo Eclesiástico de la Santa Iglesia Catedral Metropolitana, Tipografía La Unión, San Salvador, El Salvador, 1942.

Samos, R, (1986), Sobre el magisterio de Mons. Luis Chávez y González, Estudio teológico de sus cartas pastorales, Tesis de Licenciatura en Teología, Universidad Francisco Marroquín, Guatemala.

Vergara, R, y otros, (2001), Manual de Doctrina Social de la Iglesia, CELAM, Colombia. 